\title{
Luminosity distance vs. proper distance: Effects of nonlinear electrodynamics in cosmology
}

\author{
Herman J. Mosquera Cuesta ${ }^{* 1,2}$, José M. Salim ${ }^{1}$ and M. Novello ${ }^{1 \dagger}$ \\ ${ }^{1}$ Instituto de Cosmologia, Relatividade e Astrofísica (ICRA-BR) \\ Centro Brasileiro de Pesquisas Físicas, Rua Dr. Xavier Sigaud 150, Cep 22290-180, Urca, Rio \\ de Janeiro, RJ, Brazil \\ ${ }^{2}$ ICRANet International Coordinating Centre, Piazzalle della Repubblica 10, 65112, Pescara, Italy \\ Email: hermanjc@cbpf.br : : herman@icra.it
}

\begin{abstract}
By-now photons are the unique universal messengers. Cosmological sources like far-away galaxies or quasars are well-known light-emitters. Here we demonstrate that the nonlinear electrodynamics (NLED) description of photon propagation through the weak background intergalactic magnetic fields modifies in a fundamental way the cosmological redshift, $z$, that a direct computation within a specific cosmological model can abscribe to a distant source. Independently of the class of NLED Lagrangian, the effective redshift turns out to be $\left.(1+z)\right|_{\text {eff }}=(1+z) \Delta$, where $\Delta \equiv\left(1+\Phi_{e}\right) /\left(1+\Phi_{o}\right)$, with $\Phi \equiv 8 / 3\left(L_{F F} / L_{F}\right) B^{2}$, being $L_{F}=d L / d F, L_{F F}=d^{2} L / d F^{2}$, the field $F \equiv F_{\alpha \beta} F^{\alpha \beta}$, and $B$ the magnetic field strength. Thus the effective redshift is always much lower than the standard redshift, but it recovers such limit when the NLED correction $\Delta\left(\Phi_{e}, \Phi_{o}\right) \longrightarrow 1$. Therefore, once that we do not actually ever observe proper distances, then one can argue that for a particular redshift the observed luminosity distance of the light-emitting far-away source is different. The observational implications of this peculiar result are discussed.
\end{abstract}

Keywords: Cosmology: standard model :: proper distance — luminosity distance — Redshift — astronomical observables

PACS: 98.80.-k, 98.62.Ra, 95.30.Dr, 95.30.Sf

5th International School on Field Theory and Gravitation,

April 20 - 242009

Cuiabá city, Brazil

\footnotetext{
${ }^{*}$ Speaker.

$\dagger$ The author thanks Profs. Carlos Pinheiro, Gentil Pires and Alberto S. de Arruda for the invitation to deliver this lecture in the 5th ISFTG.
} 


\section{Introduction}

The study of the expansion history of the universe gained a novel dimension after the discovery of what appears to be a dimming in the luminosity emitted by supernovae type Ia (SNIa) [1], which are thought of as standard candles. These observations have been interpreted in the context of the standard cosmological model as evidence of a late-time transition from decelerate-to-acelerate expansion, according to most current viewpoints. The conclusion is attained after combining both the redshift and luminosity-distance of observed SNIa events in their Hubble diagram (HD). If one excludes any potential systematics (as for instance, there exists the possibility that we are being unable to detect, due to dust effects, much more reddened SNIa taking place at much higher redshifts (z), than close-by bluish explosions because simply these late ones are much brighter, see Ref.[2]), one can verify that their representative points in the HD appear a bit over the upper bound curve predicted by the standard Friedmann cosmology, and pile-up around $z \sim 0.5-1$, which is referred to as the transition era (see Fig. 2, below).

The redshift is the fundamental piece in achieving this conclusion, since it also enters the expression defining the luminosity distance. It is determined in almost all the cases by analysing the absorption lines from SNIa host galaxies. However, the unavoidable nonlinear interaction of light $[3,4]$ from these distant sources with the intergalactic background magnetic fields ${ }^{1}$ may crucially modify the putative value of the redshift to be abscribed to a given source from the observed lines.

On the other hand, exception done for the case in which the electric permittivity and magnetic permeability are functions of the fields, i.e., $\varepsilon(\vec{E}, \vec{B}), \mu(\vec{E}, \vec{B})$, Maxwell electrodynamics is unable to describe the nonlinear behavior of light propagation. If one follows this line of reasoning, one realizes that one way to guide ourselves to a proper investigation of the nonlinear interaction of photons [3, 4] from distant galaxies and quasars with intergalactic background fields is to keep in mind that those background magnetic fields are extremely weak! (observations rule out any electric fields, i. e., $\langle E\rangle=0$ !). Hence, if a given Lagrangian will indeed describe such photon nonlinear dynamics, it will have to depend on the invariant field $F \equiv F_{\mu v} F^{\mu v}$ in a nontrivial fashion. Interestingly, a hint to the need for a nonlinear electrodynamics (NLED) Lagrangian able to account for such dynamics came to one of us from the study of a correlated phenomenon: claims on a potential variation of the fine structure constant $\alpha$ (see [13] and references therein). Indeed, Murphy et al.[5] (Section 2.6) based on Maxwell electromagnetic theory considered large magnetic fields as a potential cause of systematic errors in their measurements of $\Delta \alpha / \alpha$. They concluded that the intra-cluster magnetic field strengths are nine orders of magnitude below the strength required to cause substantial effects. In this respect, one can state that it turns out to be easy to check that Murphy et al.'s conclusion can be reversed by considering the NLED Lagrangian density of Ref.[7].

Therefore, it appears legitimate to address the question of a potential modification of the standard cosmological redshift of light coming from far away astrophysical sources within the framework of NLED. One way to achieve this goal is to use the Lagrangian for NLED recently introduced in Ref.[7] (see also Ref.[18, 19]), whose original focus was to bring in a model based on NLED as a realization of the exotic fluid dubbed as dark energy, which is invoked to explain what is said to be the universe phase of late-time accelerate expansion. Meanwhile, we would like to stress from

\footnotetext{
${ }^{1}$ In Refs.[3, 4] have been proved that electrodynamics in a vacuum pervaded by B-fields is subject to nonlinear effects.
} 
the very beginning that the issue that shall be discussed below regarding the cosmological redshift represents an additional effect on the luminosity distance of far-away astrophysical sources caused by the evolution of this NLED cosmological background magnetic field. In this way, the analysis presented below still preserves the previous result showing that in this NLED theory the universe accelerate expansion is consistently described, although the fiducial redshift gains a small modification. Such redshift change can be interpreted as a recalibration of the transition redshift to the recent accelerate expansion.

\section{Nonlinear electromagnetism in Cosmology}

In Ref.[7] several general properties of nonlinear electrodynamics in cosmology were reviewed by assuming that the action for the electromagnetic field is that of Maxwell with an extra term, namely ${ }^{2}$

$$
S=\int \sqrt{-g}\left(-\frac{F}{4}+\frac{\gamma}{F}\right) d^{4} x
$$

where $F \equiv F_{\mu v} F^{\mu v}$. Physical motivations for bringing in this theory have been provided in Ref.[7]. Besides, a further intrinsic motivation comes from the introduction, during the last century, of both the Heisenberg-Euler and the Born-Infeld nonlinear electrodynamics, which are valid in the regime of extremely high magnetic field strengths. Both theories have been extensively investigated in the literature (see for instance $[16,17,15]$ and the long list of references therein). Since in nature non only such very strong magnetic fields do exist, then it appears to be promising to investigate also those super weak field frontiers.

Regarding Eq.(2.1), at first, one notices that for high values of the field $F$, the dynamics resembles Maxwell's one except for small corrections associate to the parameter $\gamma$, while at low strengths of $F$ it is the $1 / F$ term that dominates[8]. (Clearly, this term should dramatically affect the photon- $\vec{B}$ field interaction in intergalactic space). The consistency of this theory with observations, including the recovery of the well-stablished Coulomb law, was shown in Ref.[7], together with further analysis using the cosmic microwave radiation bound, and also after discussing the anomaly in the dynamics of Pioneer 10 spacecraft [18]. Both analysis provide small enough values for the coupling constant $\gamma$. The theory has also been successfully applied to discuss the origin of the baryon asymmetry and the amplification of primordial magnetic fields in the early universe[19].

Therefore, the electromagnetic (EM) field described by Eq.(2.1) can be taken as source in Einstein equations to obtain a toy model for the evolution of the universe which displays accelerate expansion. Such phase of acceleration runs into action when the nonlinear EM term takes over the term describing other matter fields. This NLED theory yields ordinary radiation plus a dark energy component with $w<-1$ (phantom-like dynamics). Introducing the notation ${ }^{3}$, the EM field can act as a source for the FRW model if $\left\langle E_{i}\right\rangle_{\left.\right|_{V}}=0,\left\langle B_{i}\right\rangle_{\left.\right|_{V}}=0,\left\langle E_{i} B_{j}\right\rangle_{\left.\right|_{V}}=0,\left\langle E_{i} E_{j}\right\rangle_{\left.\right|_{V}}=-\frac{1}{3} E^{2} g_{i j}$, and

\footnotetext{
${ }^{2}$ Notice that this Lagrangian is gauge invariant, and that hence charge conservation is guaranteed in this theory.

${ }^{3}$ Due to the isotropy of the spatial sections of the Friedman-Robertson-Walker (FRW) model, an average procedure is needed if electromagnetic fields are to act as a source of gravity [9]. Thus a volumetric spatial average of a quantity $X$ at the time $t$ by $\langle X\rangle_{\left.\right|_{V}} \equiv \lim _{V \rightarrow V_{0}} \frac{1}{V} \int X \sqrt{-g} d^{3} x$, where $V=\int \sqrt{-g} d^{3} x$, and $V_{0}$ is a sufficiently large time-dependent three-volume. (Here the metric sign convention (+---) applies).
} 
$\left\langle B_{i} B_{j}\right\rangle_{\left.\right|_{V}}=-\frac{1}{3} B^{2} g_{i j} .{ }^{4}$ When these conditions are fulfilled, a general nonlinear Lagrangian $L(F)$ yields the energy-momentum tensor $\left(L_{F}=d L / d F, L_{F F}=d^{2} L / d F^{2}\right)^{5}$

$$
\begin{gathered}
\left\langle T_{\mu v}\right\rangle_{\left.\right|_{V}}=(\rho+p) v_{\mu} v_{v}-p g_{\mu v}, \\
\rho=-L-4 E^{2} L_{F}, \quad p=L+\frac{4}{3}\left(E^{2}-2 B^{2}\right) L_{F},
\end{gathered}
$$

Hence, when there is only a magnetic field, the fluid can be thought of as composed of ordinary radiation with $p_{1}=\frac{1}{3} \rho_{1}$ and of another fluid with $\operatorname{EOS} p_{2}=-\frac{7}{3} \rho_{2}$. It is precisely this component with negative pressure that may drive accelerate expansion through Friedmann equations.

After presenting that theory in Ref.[7], we realized that there exists another per se equally fundamental implication of this Lagrangian. It also modifies in a significant fashion the putative redshift, $z$, or more precisely the actual luminosity distance of the source, $D_{L}=\left.r S(r)(1+z)\right|_{r_{s}}$, which then becomes different from the proper distance $\left(D_{P}=\int_{0}^{r_{s}} \sqrt{-g_{11}} d t\right)$ that one may abscribe, in the context of this toy model for cosmology, to a distant galaxy. We highlight, however, that the cosmological magnetic field is required to evolve in a particular wave to create the difference between the actual proper distance and the observed luminosity distance. These issues are discussed below.

\section{Photon dynamics in NLED: effective geometry}

Next we investigate the effects of nonlinearities in the evolution of EM waves in the vacuum permeated by background $\vec{B}$-fields. An EM wave is described onwards as the surface of discontinuity of the EM field. Extremizing the Lagrangian $L(F)$, with $F\left(A_{\mu}\right)$, with respect to the potentials $A_{\mu}$ yields the following field equation [10]

$$
\nabla_{v}\left(L_{F} F^{\mu v}\right)=0
$$

where $\nabla_{v}$ defines the covariant derivative. Besides this, we have the EM field cyclic identity

$$
\nabla_{v} F^{* \mu v}=0 \quad \Leftrightarrow \quad F_{\mu v \mid \alpha}+F_{\alpha \mu \mid v}+F_{v \alpha \mid \mu}=0 .
$$

Taking the discontinuities of the field Eq.(3.1) one gets (all the definitions introduced here are given in ${ }^{6}$ )

$$
L_{F} f_{\lambda}^{\mu} k^{\lambda}+2 L_{F F} F^{\alpha \beta} f_{\alpha \beta} F^{\mu \lambda} k_{\lambda}=0
$$

${ }^{4}$ Let us remark that since we are assuming that $\left\langle B_{i}\right\rangle_{\left.\right|_{V}}=0$, the background magnetic fields induce no directional effects in the sky, in accordance with the symmetries of the standard cosmological model.

${ }^{5}$ Under the same assumptions, the EM field associate to Maxwell Lagrangian generates the stress-energy tensor defined by Eq.(2.2) but now $\rho=3 p=\frac{1}{2}\left(E^{2}+B^{2}\right)$.

${ }^{6}$ Following Hadamard [11], the surface of discontinuity of the EM field is denoted by $\Sigma$. The field is continuous when crossing $\Sigma$, while its first derivative presents a finite discontinuity. These properties are specified as follows: $\left[F_{\mu v}\right]_{\Sigma}=0, \quad\left[F_{\mu v \mid \lambda}\right]_{\Sigma}=f_{\mu v} k_{\lambda}$, where the symbol $\left[F_{\mu v}\right]_{\Sigma}=\lim _{\delta \rightarrow 0^{+}}\left(\left.J\right|_{\Sigma+\delta}-\left.J\right|_{\Sigma-\delta}\right)$ represents the discontinuity of the arbitrary function $J$ through the surface $\Sigma$. The tensor $f_{\mu \nu}$ is called the discontinuity of the field, $k_{\lambda}=\partial_{\lambda} \Sigma$ is the propagation vector, and the symbols "|" and "॥" stand for partial and covariant derivatives. 
which together with the discontinuity of the Bianchi identity yields

$$
f_{\alpha \beta} k_{\gamma}+f_{\gamma \alpha} k_{\beta}+f_{\beta \gamma} k_{\alpha}=0
$$

A scalar relation can be obtained if we contract this equation with $k^{\gamma} F^{\alpha \beta}$, which yields

$$
\left(F^{\alpha \beta} f_{\alpha \beta} g^{\mu v}+2 F^{\mu \lambda} f_{\lambda}^{v}\right) k_{\mu} k_{v}=0 .
$$

It is straightforward to see that for Eq.(3.5) one obtains two distinct solutions: a) when $F^{\alpha \beta} f_{\alpha \beta}=0$, case in which such mode propagates along standard null geodesics, and b) when $F^{\alpha \beta} f_{\alpha \beta}=\chi$. In the case a) it is important to notice that in the absence of charge currents, this discontinuity describe the propagation of the wave front as determined by the field equation (3.1). Thence, following Lichnerowicz [12] the quantity $f^{\alpha \beta}$ can be decomposed in terms of the propagation vector $k_{\alpha}$ and a space-like vector $a_{\beta}$ (orthogonal to $k_{\alpha}$ ) that describes the wave polarization. Thus, only the light-ray having polarization and direction of propagation such that $f^{\alpha \beta} k_{\alpha} a_{\beta}=0$ will follow geodesics in $g_{\mu \nu}$. Any other light-ray will propagate on the effective metric (3.1). In this last case, we obtain from equations (3.3) and (3.5) the propagation equation for the field discontinuities being given by [6]

$$
\underbrace{\left(g^{\mu v}-4 \frac{L_{F F}}{L_{F}} F^{\mu \alpha} F_{\alpha}{ }^{v}\right)}_{\text {effective metric }} k_{\mu} k_{v}=0 .
$$

This equation proves that photons propagate following a geodesic that is not that one on the background space-time $g^{\mu v}$, but rather they follow the effective metric given by Eq.(3.6), which depends on the background field $F^{\mu \alpha}$, i. e., on the $\vec{B}$-field.

The general structure of the Lagrangian that describes the dynamics of the electromagnetic field can be represented by $L=L(F)$, where $L(F)$ is a generic functional of the Maxwell scalar $F$. The resulting theory is compatible with the covariance and gauge-preserving principles; and with the additional restrictions of the cosmological scenario adopted in our model. That Lagrangian appears as a regular function that can be developed in positive and negative powers of the invariant $F$. Positive powers dominates the dynamics of the gravitational field in the neighborhood of transients or regions of extremely high curvature, while negative powers become dominant in situations where curvature is extremely faint. It is important to notice that a similar kind of modification was already used in the Lagrangian of the gravitational field which were introduced as alternative theories to explain the late-time acceleration of the universe, see a recent review in Ref.[23]. We have generalized that idea in Refs.[7, 24] by imposing that the Lagrangian should be invariant under the map $\mathscr{X} \longrightarrow 1 / \mathscr{X}$, where $\mathscr{X}$ represents the invariant quantity that is used to construct the theory. Indeed, in the model that was introduced in [24] the Lagrangian reduces to

$$
L(F)=\alpha^{2} F^{2}-\frac{1}{4} F-\frac{\gamma}{F}+\frac{16 \alpha^{2} \gamma^{2}}{F^{2}} .
$$

The full dynamics of the model is governed by Einstein equations plus the $L(F)$ Lagrangian. It is ease to check that the first term prevents the singularity, whilst the last term in $L(F)$ prevents 
the model to allow expansion forever, that is, it constrains the magnetic field to be within the limits $B_{\max }=\frac{H_{0}}{a_{\min }^{2}}$ and $B_{\min }=\frac{H_{0}}{a_{\max }^{2}}$. For more details see Ref.[24]

Finally, if one now takes the derivative of Eq.(3.6), one can readily obtain (see in particular Ref.[18, 27] and also Refs.[15, 16, 17])

$$
k^{v} \nabla_{v} k_{\alpha}=4\left(\frac{L_{F F}}{L_{F}} F^{\mu \beta} F_{\beta}{ }^{v} k_{\mu} k_{v}\right)_{\mid \alpha} .
$$

This expression shows that the nonlinear Lagrangian introduces a term acting as a force that accelerates (positively or negatively) the photon along its path.

Thence, from all the analysis presented above it appears essential to investigate what are the effects of this peculiar photon dynamics in astrophysics and cosmology. In special, the occurrence of this phenomenon over cosmological distance scales may have a nonnegligible effect on the physical properties that one can abscribe to a given source from astronomical observables. One example of this is the cosmological redshift, i.e., the actual shifting in the position of the absorption lines from far away galaxies. ${ }^{7}$ In other words, the redshift parameter, or its associated cosmological length scale: the proper distance, gets effectively modified when computed in a standard fashion within a particular cosmological model. That is, the proper distance becomes different from the actual luminosity distance of a given far-away source.

\subsection{Effective metric as a dispersion relation for a massive photon ?}

Besides of this exceptional feature, another fundamental property of Eq.(3.6) that deserves to be discussed at this point has to do with the fact that the dispersion relation now is described by an expression of the kind $R\left(\mathrm{x}^{\mu}, \omega, \vec{k}\right)=0$, which is valid only locally. The nonlinear terms in the Lagrangian can be interpreted as describing a background medium whose main features are its dependence on space and time. Thus, such a dispersion relation would give rise to solutions of the kind $\omega=\omega\left(\mathrm{x}^{\mu}, k^{\mu}\right)$. This expression remains valid as long as the characteristic space and time scales for variations of the medium are much shorter than $k^{-1}$ and slower than $\omega^{-1}$, respectively. Aside from this, we would like to stress that our nonlinear Lagrangian does not break the gauge invariance of the electromagnetic field, and in virtue of this it is not a subject deserving to be confronted with the stringent upper bounds on the photon mass based on the assumption that a massive photon would be accompanied by an energy density of the kind $m_{\gamma}^{2} A_{\mu} A^{\mu}$, associated to the Proca field $A^{\mu}$ that describes the massive photon [26]. Moreover, the kind of modifications appearing in our dispersion relation in Eq.(3.6) is common in the presence of a space and time dependent dielectric medium where more dramatic changes to the standard dispersion relation $k_{\mu} k^{\mu}=0$ appear extensively. See for instance the exhaustive discussion provided by Mendonça in his book[27]. (See also the discussion introduced in the accompanying paper [28]).

\section{Nonlinear cosmological redshift}

\subsection{Field averaging process}

In other to be consistently called for in a cosmological setting, magnetic fields should preserve the cosmological principle, that is, to bring in no directional effects. To cope with such constraint, a

\footnotetext{
${ }^{7}$ The same theory directly leads to a variation of the fine structure constant, $\alpha[13]$
} 
proper averaging process is mandatory. To do so, let us start with the effective contravariant metric that we obtained above (see Ref.[6]), which has the form

$$
g_{\text {eff }}^{\mu \nu} \equiv L_{F} g^{\mu v}-4 L_{F F} F^{\mu \alpha} F_{\alpha}{ }^{v} .
$$

Since in our model we have

$$
F_{\mu v}=\eta_{\mu \nu}^{\alpha \beta} V_{\alpha} B_{\beta}, \quad \text { with } \quad F_{\alpha \beta} F^{\alpha \beta}=2 B^{2} .
$$

then one can now implement the average definitions for the magnetic fields introduced above, so that one can write

$$
F_{\mu \alpha} F_{v}^{\alpha}=-B_{\mu} B_{v}-B^{2} h_{\mu v}, \quad\left\langle B_{\mu} B_{v}\right\rangle=-\frac{1}{3} B^{2} h_{\mu \nu} .
$$

Thus, after the averaging procedure already indicated, the magnetic field is described by

$$
\left\langle F_{\mu \alpha} F_{v}^{\alpha}\right\rangle=-\frac{1}{3} B^{2} h_{\mu v} .
$$

One can then rewrite Eq.(4.1) explicitly in terms of the average magnetic field on the background as

$$
g_{\text {eff }}^{\mu v}=L_{F} g^{\mu v}+\frac{8}{3} L_{F F} B^{2} h^{\mu v} .
$$

On this basis, the inverse effective metric should be

$$
g_{\mu \nu}^{\mathrm{eff}}=\frac{1}{L_{F}} g_{\mu \nu}-\frac{8}{3} \frac{L_{F F} B^{2}}{L_{F}\left(L_{F}+\frac{8}{3} L_{F F} B^{2}\right)} h_{\mu \nu} .
$$

That theory leads straightforwardly to prove that the cosmological redshift of a photon traveling from a distant source to Earth is also modified, or equivalently, there is an actual change in the observed luminosity distance with respect to the proper distance of a specific far-away lightemitting cosmic source. ${ }^{8}$

\subsection{Effective cosmological redshift}

With this metric one can then compute the effective cosmological redshift since the line element provided by this NLED now reads

$$
\begin{aligned}
d s^{2} & =\frac{1}{L_{F}} d t^{2}-\left(1-\frac{8}{3} \frac{L_{F F} B^{2}}{\left[L_{F}+\frac{8}{3} B^{2} L_{F F}\right]}\right) \frac{a^{2}(t)}{L_{F}} \gamma_{i j} d \mathrm{x}^{i} d \mathrm{x}^{j} \\
& =\frac{1}{L_{F}} d t^{2}-\frac{1}{L_{F}}\left(\frac{3 L_{F}}{3 L_{F}+8 L_{F F} B^{2}}\right) a^{2}(t) d l^{2}=0 .
\end{aligned}
$$

\footnotetext{
${ }^{8}$ In any case, irrespective of the magnetic field cosmological evolution, such NLED effects will become evident only for redshifts smaller than the one identified as the transition era $2<z<1$. In other words, for fields such that the term $B^{4}$ is much greater than the coupling constant $\gamma$ the contribution of this theory through the factor $\Delta$ in Eq.(4.8) becomes negligible. That is, nothing changes in the universe earlier evolution. This conclusion is in agreement with what was stated in Ref.[7].
} 


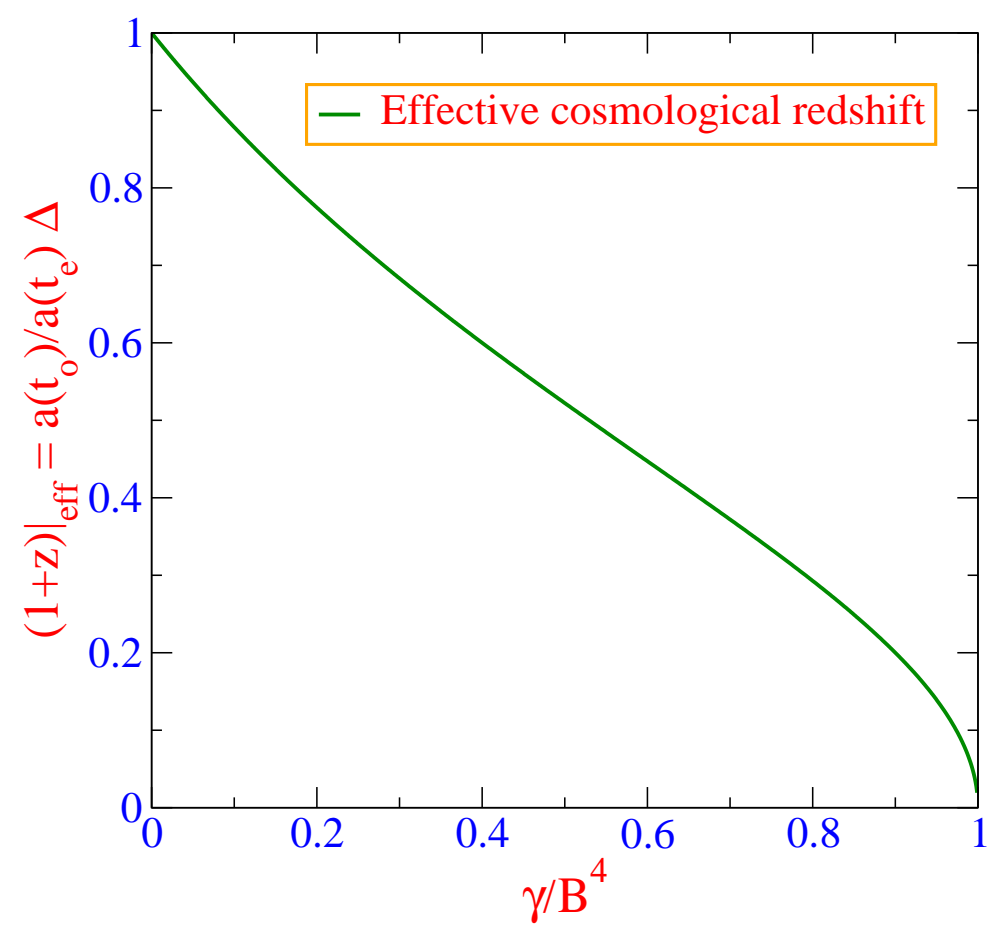

Figure 1: The effective cosmological redshift $\left.(1+z)\right|_{\mathrm{eff}}=\frac{a\left(t_{2}\right)}{a\left(t_{1}\right)} \Delta$ as a function of the intergalactic $B$-field strength normalized as $\gamma / B^{4}$, after the NLED correction to the standard cosmological redshift $(1+z)=$ $\frac{a\left(t_{2}\right)}{a\left(t_{1}\right)}$, (here we identify: $t_{1}=t_{e}$, and $t_{2}=t_{0}$ ). The resulting redshift mimics a cosmological model that has $\Omega_{m}=0.27$, as indicated by Eqs.(1,2), when indeed it is built from a Friedmann-Lemaître-Robertson-Walker model which has $\Omega_{m}=1$. Notice, however, that the effective redshift does not reach the limit $z=0$, i.e., the $\gamma / B^{4}=1$ limit is not attainable, as argumented in the text.

By identifying the effective metric from Eq.(4.7), and computing the $g_{00}$ component, it follows then that the expression for the cosmological redshift turns out to be

$$
\left.(1+z)\right|_{\mathrm{eff}} \equiv \frac{c \delta t_{0}}{c \delta t_{e}}=\frac{a\left(t_{0}\right)}{a\left(t_{e}\right)} \Delta=(1+z) \Delta,
$$

where $\Delta \equiv\left[\left.(1+\Phi)^{1 / 2}\right|_{t_{e}}\right] /\left[\left.(1+\Phi)^{1 / 2}\right|_{t_{0}}\right]$, and $\Phi \equiv 8 / 3\left(L_{F F} / L_{F}\right) B^{2}$. The specific modification of the redshift depends on the particular problem we focus on, in particular, a similar effect was already analyzed in the presence of very strong magnetic fields in pulsars [15, 16, 17].

As an example, in the case of cosmology, as pointed out above, a model to explain the recently discovered late acceleration of the universe by using a NLED described by the Lagrangian $L(F)=$ $-\frac{1}{4} F+\frac{\gamma}{F}$, where $F \equiv F_{\alpha \beta} F^{\alpha \beta}$ and $\gamma=-v^{2}$, was proposed in Ref.[7]. Thus, by using Eqs.(4.6, 4.8), one can compute the actual redshift of a given cosmic source.

Therefore, the cosmological redshift turns out to be

$$
\left.(1+z)\right|_{\mathrm{eff}}=\frac{c \delta t_{0}}{c \delta t_{e}}=\frac{a\left(t_{0}\right)}{a\left(t_{e}\right)} \frac{\left.\left(\frac{B^{4}+\frac{5}{3} v^{2}}{B^{4}-v^{2}}\right)^{1 / 2}\right|_{t_{e}}}{\left.\left(\frac{B^{4}+\frac{5}{3} v^{2}}{B^{4}-v^{2}}\right)^{1 / 2}\right|_{t_{0}}}
$$


With this equation one can plot the effective cosmological redshift according to NLED for a specific field strength $B$. That result is presented in Fig.-1, which illustrates, through the Hubble diagrams presented in Fig.-2, a noticeable effect on the redshift. After analyzing Fig.-1, one realizes that the first direct implication of this result is that the determination, from direct observations, of the redshift of a given distant quasar or galaxy may lead to a mistaken interpretation on the actual luminosity distance to those sources if such NLED effect is not properly accounted for. Such a task could be performed by estimating the angular-average of the quadratic $B$-field over the intergalactic space.

\section{Discussion and conclusion}

Our main result is presented in Eq.(4.8). Irrespective of the structure of the Lagrangian, it is valid for any generic Lagrangian $L(F)$ describing a NLED theory, provided the field averaging procedure indicated earlier in Eq.(4.4) is held. Therefore, in order to properly address the issue on the actual luminosity distance, or cosmological redshift, of a distant astrophysical object this NLED effect should be first taken into account. In the specific case of the model introduced in Ref.[7] to provide a model of dark energy based on NLED to explain the currently purported idea that our universe is undergoing a late-time acceleration phase, the above Eq.(4.8) becomes Eq.(4.9) which shows the dependence of the NLED effect on the relation between the intervening intergalactic space $B$-field strength and the coupling constant of the theory, $\gamma$, as shown in Fig.-1.

By looking at Fig.-1, and comparing to the standard calculation within a given model, viz., one must address to the model introduced in Ref.[7], one notices that for values of the ratio $|\gamma| / B^{4} \longrightarrow 1$, one obtains much lower redshifts than the standard cosmological redshift $1+z$, which is recovered in the limit $B^{4}>>\gamma$. Therefrom, and cosmologically crudely speaking, in any NLED theory the proper distance: $D_{P}=\int_{0}^{r_{s}} \sqrt{-g_{11}} d t$, of the light-emitting far-away source at $r_{s}$ is different from its actual luminosity distance: $D_{L}=\left.\left.r S(r)(1+z)\right|_{\mathrm{eff}}\right|_{r_{s}}$.

Aside from all that, Eq.(4.8) makes it evident that the NLED correction is already "built-in" in the actual luminosity distance, or redshift of the distant source, estimated for instance from the SN host-galaxy absorption lines. In other words, after estimating the $B$-field strength of the intervening intergalactic space of each of the already observed SNIa, (typically $10^{-11} \mathrm{G}$ for compatibility with the $\Lambda$-CDM model predictions for structure formation [31,30], while for galaxies is found $10^{-6}$ $10^{-8} \mathrm{G}$ ), the actual redshift to be plotted in the Hubble diagram $(\mu(z)$ vs. $z)$ is going to be the effective redshift $\left.(1+z)\right|_{\text {eff }}$, i.e. the one directly estimated from the observations, after discounting the correction factor $\Delta$ provided by NLED (see illustrative examples in plots c, $\mathrm{d}$ in Fig.-2).

From this analysis one concludes that in case the NLED theory for the photon interaction (in a vacuum) with extragalactic background magnetic fields be realized in nature, it would become evident that any conclusion on actual cosmological redshifts drawn from SNIa or/and observations in the optical of GRBs afterglows had to be revised. In any case, any general NLED theory will lead to an effective cosmic redshfit $\left.(1+z)\right|_{\text {eff }}=(1+z) \Delta$. In the specific case of $L(F)=F+1 / F$, wondering whether the theory has something to do with nature rests on future experiments and/or observations like observing absorption lines in both a supernova and a gamma-ray burst afterglow in SN/GRB related events, a connection that is by now conclusively demonstrated for a number of cases [25]. 

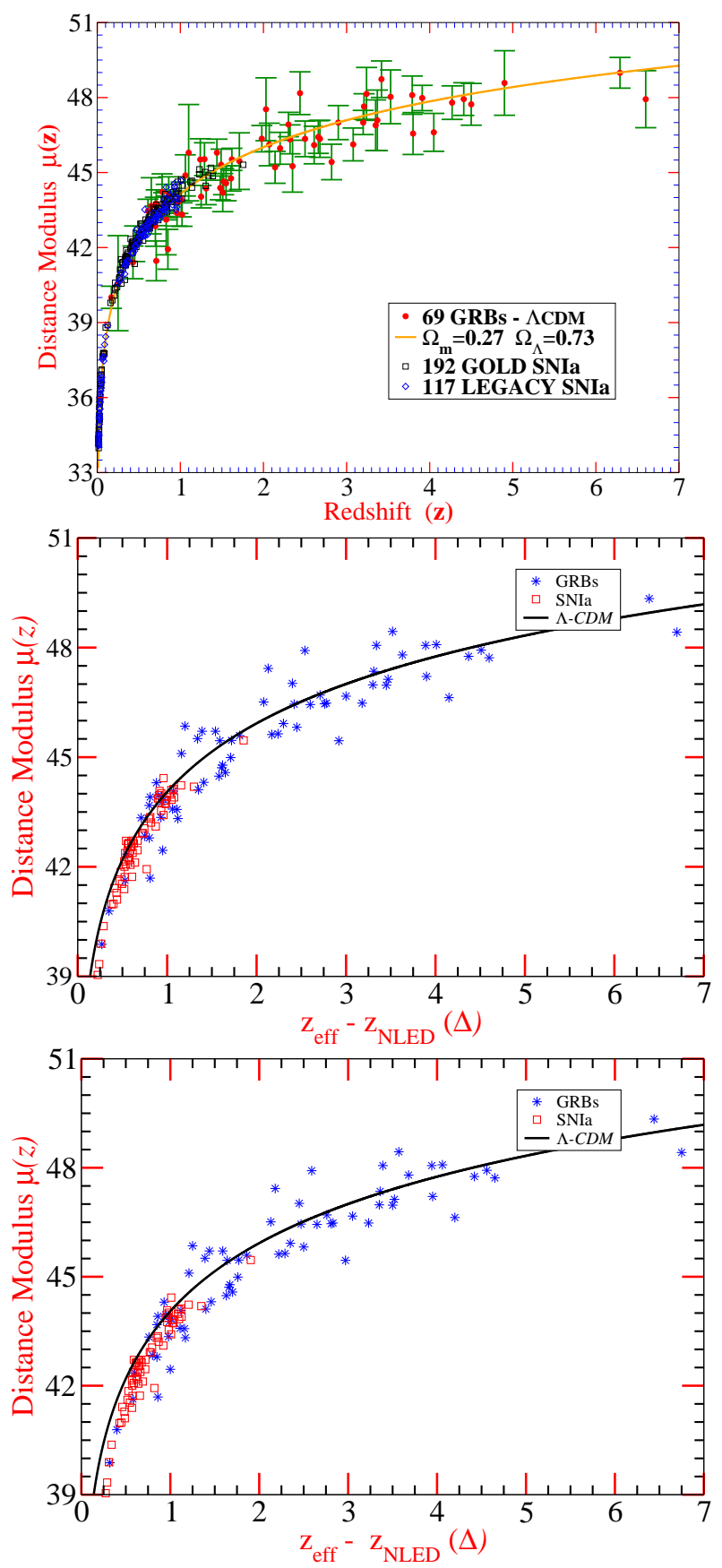

Figure 2: Hubble diagram of the 69 GRBs sample (red filled circles) of Ref.[21] calibrated for $\Lambda$-CDM, 192 GOLD SNIa (blue squares) and 117 LEGACY SNIa (black diamonds) samples of Refs.[1, 22], as current observations indicate (no NLED correction included). One may conjecture that most, if not all, of the events presenting a much higher luminosity in this plot could have taken place in sources where the local $B$-field is near the critical one defined from $\gamma$. The next two graphs illustrate SNIa, GRBs global shifts of 0.1, 0.15 in $z$ (no error bars included). They are intended here solely to illustrate the overall effect. The attentive reader should bear in mind that the NLED correction should indeed be applied to each individual SNIa or GRB event, once one has estimated the intervening (local) host-galaxy $B$-field through Zeeman splitting, cosmic rays deflection; i.e. correlation of the direction of such particles with the direction of the emitter, or some other astrophysical technique. This analysis will be presented elsewhere. 
Acknowledgements: HJMC thanks Prof. R. Ruffini and ICRANet Coordinating Centre, Pescara, Italy, for hospitality during the preparation of this paper. HJMC is a fellow of Fundação de amparo à Pesquisa do Estado do Rio de Janeiro (FAPERJ)

\section{References}

[1] S. Perlmutter, et al., Nature 392, 311 (1998); A. Riess, et al., Astron. J. 116, 1009 (1998), S. Perlmutter, et al., Astrophys. J. 517, 565 (1999), J. Tonry, et al., Astrophys. J. 594, 1 (2003); P. Astier, et al., Astron. \& Astrophys. 447, 31 (2006)

[2] F. Mannucci, M. della Valle, N. Panagia, How many supernovae are we missing at high redshift?, MNRAS 377, 1229 (2007). e-print astro-ph/0702355

[3] D. L. Burke et al., Phys. Rev. Lett. 79, 1626 (1997)

[4] M. Marklund, P. Shukla, Rev. Mod. Phys. 78, 591-640 (2006)

[5] M. T. Murphy et al., MNRAS 327, 1223 (2001)

[6] M. Novello, et al. Phys. Rev. D 61, 045001 (2000)

[7] M. Novello, S. E. Pérez Bergliaffa and J. M. Salim, Phys. Rev. D 69, 127301 (2004)

[8] Notice that on dimensional grounds, $\gamma=\hbar^{2} \times v^{2}$, where $v$ is a fundamental constant with dimension of length $^{-4}$.

[9] R. Tolman and P. Ehrenfest, Phys. Rev. 36, 1791 (1930).

[10] J. Plebanski, Lectures in nonlinear electrodynamics (Nordita, Copenhagen, 1970)

[11] J. Hadamard, Leçons sur la propagation des ondes et les equations de l'Hydrodynamique (Hermann, Paris, 1903)

[12] A. Lichnerowicz, Elements of Tensor Calculus, (John Wiley and Sons, New York, 1962). See also Relativistic Hydrodynamics and Magnetohydrodynamics (W. A. Benjamin, 1967), and Magnetohydrodynamics: Waves and Shock Waves in Curved Space-Time (Kluwer, Springer, 1994)

[13] J.-P. Mbelek and H. J. Mosquera Cuesta, MNRAS 389, 199-204 (2008). e-print: arXiv astro-ph/0707.3288

[14] V. A. de Lorenci, et al., Phys. Lett. B 482, 134 (2000)

[15] H. J. Mosquera Cuesta, José A. de Freitas Pacheco, José M. Salim, Int. J. Mod. Phys. A 21:43-55 (2006)

[16] H. J. Mosquera Cuesta, José M. Salim, Mon. Not. Roy. Ast. Soc. 354: L55-L59 (2004)

[17] H. J. Mosquera Cuesta, José M. Salim, Astrophys. J. 608:925-929 (2004)

[18] J.-P. Mbelek, H. J. Mosquera Cuesta, M. Novello and J. M. Salim, Eur. Phys. Lett. 77, 19001 (2007)

[19] H. J. Mosquera Cuesta and G. Lambiase, Phys. Rev. D 80, 023013 (2009)

[20] J. P. Vallée, New Astron. Rev. 48, 763 (2004)

[21] Schaefer B. E., Astrophys. J. 660, 16 (2007)

[22] L. Balázs, et al., Astronom. Nachricht. 327, Issue 9, 917 (2006)

[23] Thomas P. Sotiriou, Stefano Liberati, Metric-affine $f(R)$ theories of gravity, Annals Phys. 322:935-966 (2007). e-Print: gr-qc/0604006 
[24] M. Novello, et al., Rebouncing in a cycling universe, arXiv:0802.1875v1 [astro-ph]

[25] J. S. Bloom and S. E. Woosley, The supernova-gamma-ray burst connection, Ann. Rev. Astron. Astrophys. 44: 507-556 (2006)

[26] E. Adelberger, G. Dvali, A. Gruzinov, Phys. Rev. Lett. 98, 010402 (2007)

[27] J. T. Mendonça, Theory of photon acceleration, (Institute of Physics Publishing, Bristol and Philadelphia 2001)

[28] H. J. Mosquera Cuesta, L'energie sombre: Un mirage cosmique? Luminosity distance $\neq$ proper distance: A cosmological dissimilitude induced by nonlinear electrodynamics, in Proceedings of the International Conference Invisible Universe, Palais de l'UNESCO, Paris, France (2009)

[29] K. Ichiki, et al., Science 311, 827 (2006)

[30] E. Waxman, P. Coppi, Astrophys. J. 464, L75 (1996). See also, E. Waxman, J. Miralda-Escudé, Astrophys. J. 472, L89 (1996)

[31] J. P. Vallée, Astrophys. J. 360, 1-6 (1990); Astron. and Astrophys. 239, 57-62 (1990); Astronom. J. 99, 459-462 (1990) 